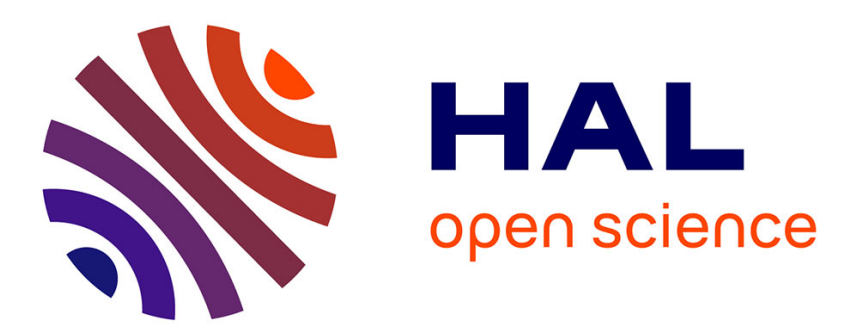

\title{
Hyperbolic Wavelet Transform for Historic Photographic Paper Classification Challenge
}

Stéphane Roux, Patrice Abry, Herwig Wendt, Stéphane Jaffard, Béatrice Vedel

\section{- To cite this version:}

Stéphane Roux, Patrice Abry, Herwig Wendt, Stéphane Jaffard, Béatrice Vedel. Hyperbolic Wavelet Transform for Historic Photographic Paper Classification Challenge. 48th Annual Asilomar Conference on Signals, Systems, and Computers (ASILOMAR 2014), Nov 2014, Pacific Grove, United States. pp. 1-5. hal-01394661

\section{HAL Id: hal-01394661 \\ https://hal.science/hal-01394661}

Submitted on 9 Nov 2016

HAL is a multi-disciplinary open access archive for the deposit and dissemination of scientific research documents, whether they are published or not. The documents may come from teaching and research institutions in France or abroad, or from public or private research centers.
L'archive ouverte pluridisciplinaire HAL, est destinée au dépôt et à la diffusion de documents scientifiques de niveau recherche, publiés ou non, émanant des établissements d'enseignement et de recherche français ou étrangers, des laboratoires publics ou privés. 


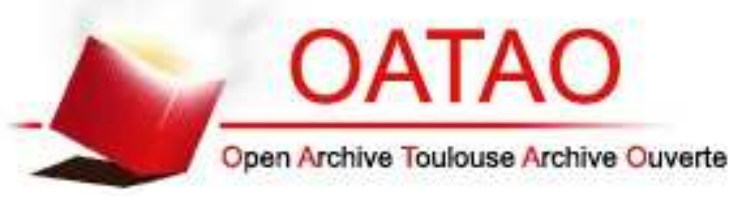

\section{Open Archive TOULOUSE Archive Ouverte (OATAO)}

OATAO is an open access repository that collects the work of Toulouse researchers and makes it freely available over the web where possible.

This is an author-deposited version published in : http://oatao.univ-toulouse.fr/ Eprints ID : 15186

The contribution was presented at ASILOMAR 2014:

http://www.asilomarsscconf.org/

To cite this version : Roux, Stéphane and Abry, Patrice and Wendt, Herwig and Jaffard, Stéphane and Vedel, Béatrice Hyperbolic Wavelet Transform for Historic Photographic Paper Classification Challenge. (2015) In: 48th Annual Asilomar Conference on Signals, Systems, and Computers (ASILOMAR 2014), 2 November 2014 - 5 November 2014 (Pacific Grove, United States).

Any correspondence concerning this service should be sent to the repository administrator: staff-oatao@listes-diff.inp-toulouse.fr 


\section{Hyperbolic Wavelet Transform for Historic Photographic Paper Classification Challenge}

\author{
S. Roux, P. Abry \\ CNRS, Physics Dept., \\ ENS Lyon, France, \\ patrice.abry@ens-lyon.fr
}

\author{
H. Wendt \\ CNRS, IRIT \\ University of Toulouse, \\ France
}

\author{
S. Jaffard \\ CNRS, LAMA, \\ Paris Est Univ., \\ Créteil, France
}

\author{
B. Vedel \\ LMAM, \\ Univ. Bretagne Sud, \\ Vannes, France
}

\begin{abstract}
Photographic paper texture characterization constitutes a challenging image processing task and an important stake both for manufacturers and art museums. The present contribution shows how the Hyperbolic Wavelet Transform, thanks to its joint multiscale and anisotropic nature, permits to achieve an accurate photographic paper texture analysis and characterization. A cepstral-type distance, constructed on the coefficients of the Hyperbolic Wavelet Transform, is then used to measure similarity between pairs of paper textures. Spectral clustering followed by Ascendant Hierarchical Clustering applied to the similarity matrix enables an unsupervised classification of photographic paper sheets. This methodology is applied to a test dataset made available in the framework of the Historic Photographic Paper Classification Challenge. The relevance of the proposed texture characterization and classification procedure is assessed by comparisons against the database documentation provided by experts.
\end{abstract}

\section{INTRODUCTION}

Historic Photographic Paper Classification. There has recently been a growing interest for technical art history and investigations of the potential benefits of signal and image processing tools for addressing issues of interest to art experts. The study of historical photographic paper constitutes one prominent example where image processing tools aiming at texture characterization might prove able to permit performing tasks such as photographic paper and photography classification automatically. Paper surface texture is indeed a major property for printed art photography, revealing the aesthetic intentions of the artist and impacting the public perception. Photographic paper texture characterization has thus received significant research efforts, as it is crucial for both art experts in museums and manufacturers. Indeed, texture characterization may potentially enable art experts to identify fake prints or to detect systematic preferences or atypical choices within an artist's body of work [17], [18]. Texture analysis also starts to be applied to inkjet photographic materials [19].

To date, art experts mostly assess unknown textures by visually or manually comparing it with known reference textures, which turns into a time consuming and tedious task for datasets of large size. There is thus an obvious need for automated and systematic texture analysis procedures assisting experts in art photo paper classification. In that context, at the initiative of Paul Messier, a world-renowned paper conservator (http://paulmessier.com/) and of the Museum of Modern Art (NYC), a Historic Photographic
Paper Classification (HPPC) Challenge has been organized, with a test dataset made publicly available for analysis (http://www.papertextureid.org/). This dataset will be used in the present contribution.

Texture Analysis. Texture analysis is a classical task in image processing that received a considerable amount of research efforts over the past 20 years, cf. e.g., [6], [9], [10], [23], [24]. Texture analysis has also, much more recently, been used for art investigations, though only to a much lesser extent than for other applications (cf. e.g., [1]-[5], [20] and references therein). Amongst the many paradigms that have been used to characterize textures, the fractal - or scale invariance - paradigm is receiving increasing attention, notably in biomedical contexts [14], [15], in physics of surfaces and fractures [16], and in geophysics [21]. The fractal paradigm has also been extended to multidimensional multifractal analysis of textures (notably, for images), cf. e.g., [3], [12], [26]. However, most of these attempts do not take into account the potential anisotropy of the texture. Recently, it has been proposed to specifically include anisotropy in scaling analysis of textures [22] by making use of the Hyperbolic Wavelet Transform (HWT) [7].

Goals, contributions and outline. Elaborating on [13], the present contribution applies the anisotropic multiscale texture analysis tools developed in [22] (cf. Section III-A) to the characterization of the art photography paper dataset, made available in the context of the HPPC Challenge, and described in Section II. From the HWT coefficients, features are extracted and cepstral distances between features from different textures are computed (cf. Section III-B). The classification approach applied to these distances consists of a standard Spectral Clustering procedure (cf. e.g., [8], [11], [25]), briefly sketched in Section III-C. Results in terms of distances computed from the HWT representation and of the achieved clustering are discussed and interpreted in Section IV.

\section{DATA SET}

The art photography paper reference dataset, made publicly available in the framework of the HPPC Challenge at papertextureid.org, has been constructed for pedagogical purposes and is organized in 12 subsets (of 10 samples 
each), representing a variety of typical photographic papers of different types and produced by different manufacturers. Within these subsets, there are three levels of similarity: Subset1 to 3 consist of samples from one same sheet; Subset 4 to 6 consist of samples from different sheets yet taken from the same manufacturer package of sheets; Subset7 to 9 consist of samples from papers made to the same manufacturer specifications over a period of time. Furthermore, Subset10 to 12 consists of 30 additional sheets of interest to art experts. This reference dataset has been carefully documented by an art expert, P. Messier, providing metadata such as texture, reflectance, manufacturer, brand and date for each sheet in the database, cf., [13] for further details.

Samples are obtained from a raking light imaging technique, designed by P. Messier, and described in [13]. They consist of $1.00 \times 1.35 \mathrm{~cm}^{2}$ of photographic paper surface, digitized at $153.6 \mathrm{pixel} / \mathrm{mm}$, resulting in $1536 \times 2080$ images, with each pixel thus corresponding to $6.51^{2}=42.4 \mu \mathrm{m}^{2}$. Examples are plotted in Fig. 1.
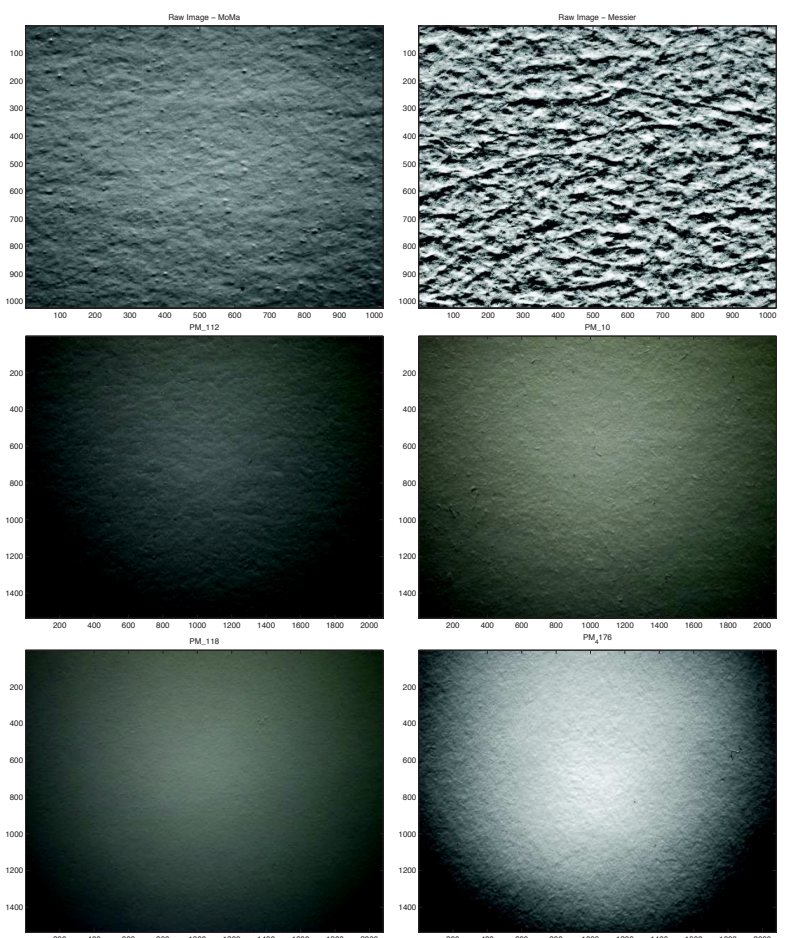

Figure 1. Art photographic paper textures. Examples of raking light photographic paper samples.

\section{Methodology}

\section{A. Features: Hyperbolic Wavelet Transform}

Hyperbolic Wavelet Transform. The Hyperbolic Wavelet Transform (HWT) [7] consists of one of the numerous declination of image multiscale (or multiresolution) analysis. Let $\psi$ be a mother wavelet, characterized by its uniform regularity index and by its number of vanishing moments $N_{\psi}$. The latter is a positive integer, defined as $\forall n=0, \ldots, N_{\psi}-1$,
$\int_{\mathcal{R}} t^{k} \psi(t) d t \equiv 0$ and $\int_{\mathcal{R}} t^{N_{\psi}} \psi(t) d t \neq 0$. From $\psi$, a basis of $L^{2}\left(\mathbb{R}^{2}\right)$ is constructed as the following tensor products:

$$
\begin{aligned}
& \psi_{j_{1}, j_{2}, k_{1}, k_{2}}\left(x_{1}, x_{2}\right)= 2^{-\left(j_{1}+j_{2}\right)} \\
& \cdot \psi\left(2^{-j_{1}} x_{1}-k_{1}\right) \psi\left(2^{-j_{2}} x_{2}-k_{2}\right), \\
& \psi_{0, j_{2}, k_{1}, k_{2}}\left(x_{1}, x_{2}\right)= 2^{-j_{2}} \varphi\left(x_{1}-k_{1}\right) \psi\left(2^{-j_{2}} x_{2}-k_{2}\right), \\
& \psi_{j_{1}, 0, k_{1}, k_{2}}\left(x_{1}, x_{2}\right)=2^{-j_{1}} \psi\left(2^{-j_{1}} x_{1}-k_{1}\right) \varphi\left(x_{2}-k_{2}\right), \\
& \psi_{0,0, k_{1}, k_{2}}\left(x_{1}, x_{2}\right)=\varphi\left(x_{1}-k_{1}\right) \varphi\left(x_{2}-k_{2}\right),
\end{aligned}
$$

The coefficients of the HWT are obtained by scalar product of the texture $X\left(x_{1}, x_{2}\right)$ with the collection of functions $\psi_{(\cdot)}$ defined above, for different dilation and translation factors:

$$
\begin{array}{r}
T_{X}\left(\left(j_{1}, j_{2}\right),\left(k_{1}, k_{2}\right)\right)=\left\langle X\left(x_{1}, x_{2}\right), \psi_{j_{1}, j_{2}, k_{1}, k_{2}}\left(x_{1}, x_{2}\right)\right\rangle, \\
\forall j_{1} \geq 0, j_{2} \geq 0 .
\end{array}
$$

HWT is thus practically computed by repeating iteratively a two-step procedure, consisting first of performing a 2D-DWT and second to refine the scale decomposition (cf. Fig. 2).
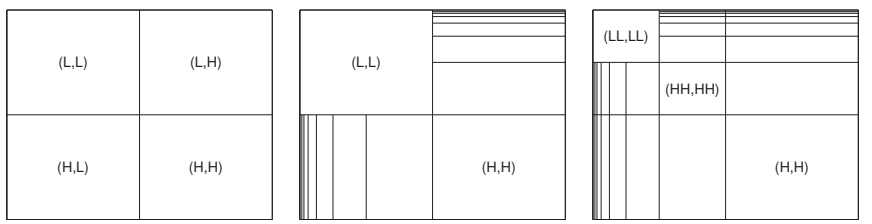

Figure 2. HWT algorithm. The HWT algorithm consists of first performing a 2D-DWT transform (left) followed by further refining the scale decomposition (middle) and iterating the procedure (right).

Features: multiscale representations. The proposed multiscale representation is obtained by computing space averages of the $q$ th power of $T_{X}\left(\left(j_{1}, j_{2}\right),\left(k_{1}, k_{2}\right)\right)$ at fixed scales:

$$
S_{X}\left(\left(j_{1}, j_{2}\right), q\right)=\frac{1}{n_{a}} \sum_{\underline{k}}\left|T_{X}\left(\left(j_{1}, j_{2}\right),\left(k_{1}, k_{2}\right)\right)\right|^{q}, q>0 .
$$

An example of such an anisotropic multiscale representation is plotted in Fig. 3. The use of the classical 2D discrete wavelet transform (2D-DWT), which relies on a single and same dilation factor for both horizontal and vertical axes $2^{j_{1}} \equiv 2^{j_{2}}$, would correspond to compute only $S_{X}((j, j), q)$ (marked as the black dashed diagonal in Fig. 3, right plot). In contradistinction, the HWT makes use of different dilation factors along each direction (corresponding to varying the direction of the black solid line in Fig. 3, right plot), yielding a richer information than $S_{X}((j, j), q)$ only and thus permitting to capture potential anisotropies in textures, be they associated to scaling properties or not [22]. The function $S_{X}\left(\left(j_{1}, j_{2}\right), q\right)$ computed for $q=2$ and a collection of analysis scales ranging from $13 \mu m \leq 2^{j_{1}}, 2^{j_{2}} \leq 830 \mu m \equiv 0.83 m m$ (i.e. 7 octaves) will constitute the $7 \times 7=49$ features that will be used below for textures characterization and classification.

\section{B. Distances}

Proximity between textures $X$ and $Y$ is measured via a cepstral-type distance between the multiscale representations 

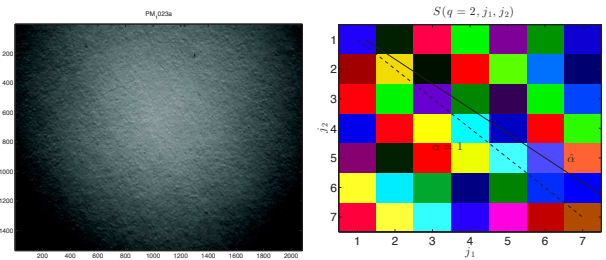

Figure 3. HWT analysis example. Sample photographic paper texture with its HWT-based anisotropic multiscale representation.

$S_{X}\left(\left(j_{1}, j_{2}\right), q\right)$ and $S_{Y}\left(\left(j_{1}, j_{2}\right), q\right)$. It consists of a classical $L^{p}$ norm computed on $S_{X}\left(\left(j_{1}, j_{2}\right), q\right)$ after log-transformation and normalization (with $p>0$ ):

$$
d(X, Y)=\left(\sum_{j_{1}, j_{2}}\left|\tilde{S}_{X}\left(2^{j_{1}}, 2^{j_{2}}, q\right)-\tilde{S}_{Y}\left(2^{j_{1}}, 2^{j_{2}}, q\right)\right|^{p}\right)^{\frac{1}{p}},
$$

where

$$
\tilde{S}_{X}\left(2^{j_{1}}, 2^{j_{2}}, q\right)=\ln \frac{S_{X}\left(2^{j_{1}}, 2^{j_{2}}, q\right)}{\sum_{j_{1}^{\prime}, j_{2}^{\prime}} S_{X}\left(2^{j_{1}^{\prime}}, 2^{j_{2}^{\prime}}, q\right)} .
$$

The normalization of $\tilde{S}_{X}$ ensures robustness against change of light intensity. The log-transformation (cepstral measure) ensures that all scales contribute to the distance as it is experimentally observed that the multiscale representation varies across scales roughly as a power law: $S_{X}(\alpha j,(2-\alpha) j, 2) \simeq$ $C_{\alpha} 2^{j H_{\alpha}}$

In the present work, $q=2$ and $p=1$ are used, and no specific attempt for optimal tuning of these parameters to improve classification performance has been undertaken.

\section{Spectral clustering}

Spectral clustering is used as a non-supervised classification procedure aiming to reduce the dimensionality of the space in which samples are represented to ensure robustness of the classification, cf. e.g., [8], [11]. Starting from the $N \times N$ cepstral distance matrix $d$, where $N$ is the number of photographic paper samples, it consists in: (1) transforming the cepstral-type distances $d(X, Y)$ into a (dis)similarity matrix $\mathcal{D}=\exp (-d / \epsilon)$, where $\epsilon$ is a constant assessing the typical closeness between images; (2) computing the eigenvalues and eigenvectors of the Laplacian operator associated to $\mathcal{D}, \mathscr{L}=$ $I-D^{-1 / 2} \mathcal{D} D^{-1 / 2}$, where $D=\operatorname{diag}\left(\sum_{j} \mathcal{D}_{i j}\right)$ is the diagonal matrix of the strengths $\sum_{j} \mathcal{D}_{i j}$ ); (3) sorting the eigenvalues of $\mathscr{L}$ by decreasing order and truncating the expansion at order $K$; (4) performing Ascending Hierarchical Clustering of the $K \times N$ matrix consisting of the $K$ first eigenvectors as the set of coordinates (of reduced dimensionality $K \ll N$ ) for the $N$ photo paper samples, and thresholding distances so as to obtain $K$ clusters.

\section{RESULTS AND DISCUSSION}

\section{A. Distances}

As a preliminary investigation, let us start by comparing two different sub-pieces of one same sample of photographic paper.
Fig. 4 compares the multiscale representation $S_{X}\left(\left(j_{1}, j_{2}\right), 2\right)$ computed on the entire samples and those obtained from two sub-pieces. It clearly shows that the two sub-pieces have quasiidentical representations thus showing the spatial homogeneity of the samples and hence the relevance of computing $S_{X}\left(\left(j_{1}, j_{2}\right), 2\right)$ across the entire sample.
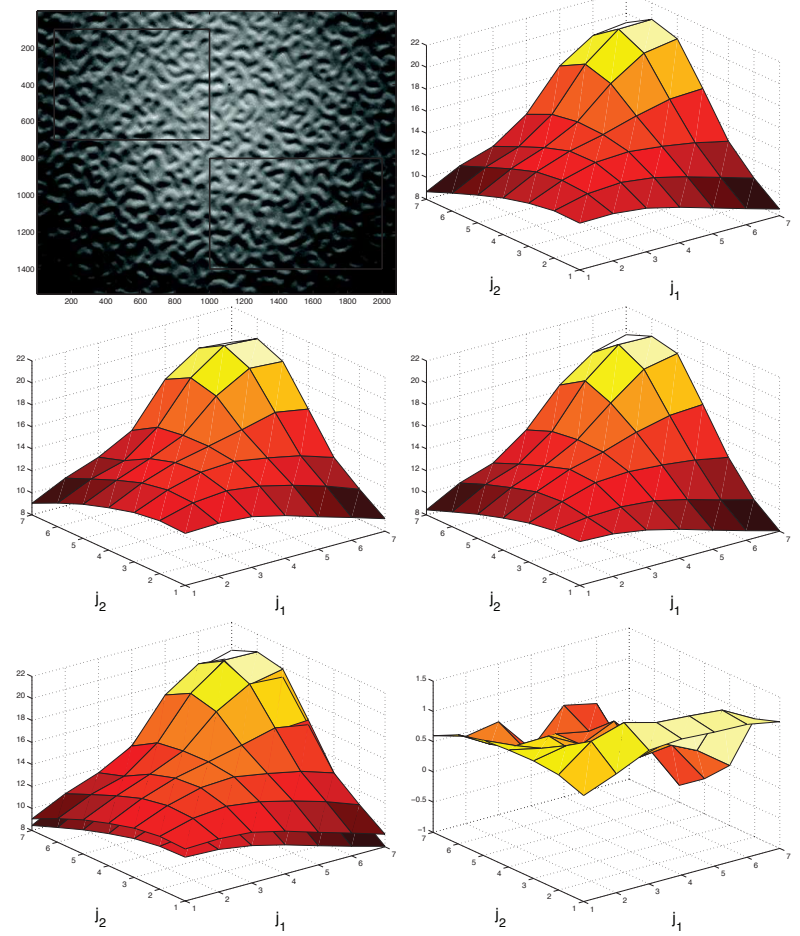

Figure 4. Multiscale representation within sample. Top row: Sample photographic paper and multiscale representation $S_{X}\left(\left(j_{1}, j_{2}\right), 2\right)$. Middle row: multiscale representations computed from the two sub-pieces of the sample marked with black rectangles. Bottom row: superposition and difference of these two multiscale representations, respectively.

As a second preliminary investigation, the experts in charge of the HPPC Challenge questioned whether the direction of the raking light may impact analysis or not. Fig. 4 compares the multiscale representations $S_{X}\left(\left(j_{1}, j_{2}\right), 2\right)$ computed for one same sample using raking light along two different directions (North and North-East). Despite the visual diagonal roughness structure visible on the image receiving light from Northeast, the multiscale representations are quasi-identical, which demonstrates the independence of the representation from the light direction.

To illustrate the relevance and effectiveness of the proposed multiscale representation and cepstral distance for texture characterization, let us compute the median intra- and interdistances for the 10 a priori known clusters $(3 \times 3$ of 10 samples each, and 1 cluster for the 30 last samples). Fig. 6 (left) plots the distances between each cluster and clearly shows a black diagonal (for the 9 first clusters) indicating, as expected, much lower intra-cluster distances than inter-cluster distances. It also shows elementary results, such as the fact that Subset2 (samples from same sheet) and Subset4 (samples from same 

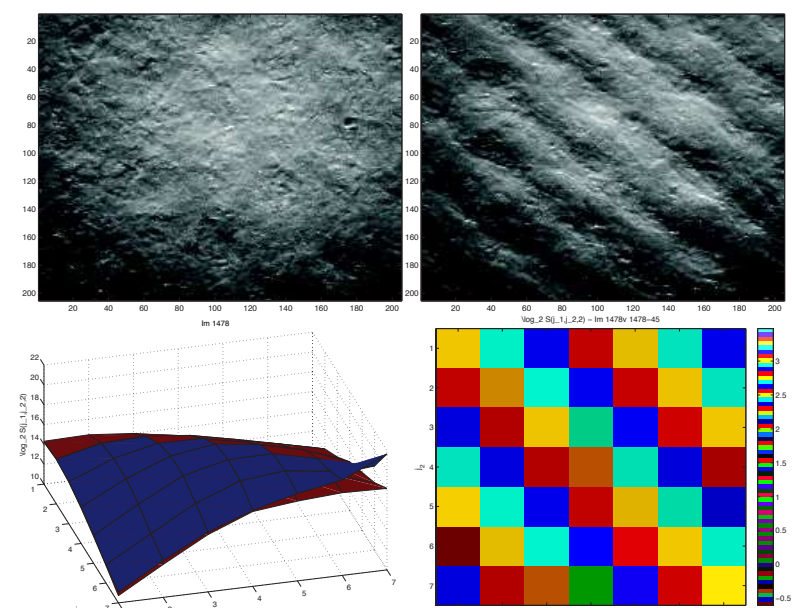

Figure 5. Raking light directions. Top row: one same sample receiving light along two different directions (North and North-east). Bottom row: Superimposition and difference of the multiscale representation $S_{X}\left(\left(j_{1}, j_{2}\right), 2\right)$ computed from the sample illuminated along two directions.

packet) have a low inter-cluster distance, indicating that the sheet from Subset2 likely belong to the packet in Subset4, or is very closely related to it. The same holds for Subsets5 and 7, suggesting that the packet in Subset5 has likely been produced by the Manufacturer of Subset7. More interestingly, since not expected by art experts, Fig. 6 (right) displays the intra-cluster distances (diagonal of right plot) and shows that median distances (as well as maximum absolute deviations) between samples from different sheets from one same packet (Subsets4-6) are not larger than median distances between samples from one same sheet (Subsets1-3). This indicates a perfect reproducibility in the manufacturing process for all the sheets of a single packet. These intra-subset median distances increase for samples from same manufacturers but different sheet and packet, Subset7-9.
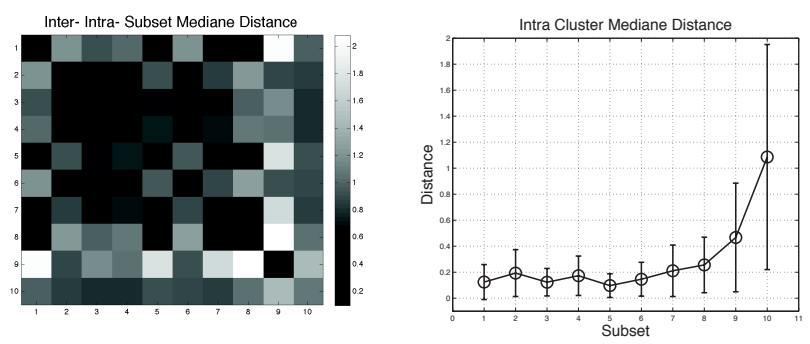

Figure 6. Distances for a priori known Subsets. Left: matrix of intra (diagonal) and inter (off-diagonal distance). Right: Evolution of the intracluster median distance for samples from the same sheet (1-3), samples from different sheets from the same packet (4-6), samples from same manufacturers but sheets from different packets, Subset7-9.

\section{B. Clustering}

In the spectral clustering procedure described in Section III-C above, the choice of the relevant number of clusters $K$ remains to be set empirically. A practical rule of thumb consists in plotting the eigenvalues in ascending order together with their successive differences: the local maxima of the differences correspond to values of $K$ where the clusters are
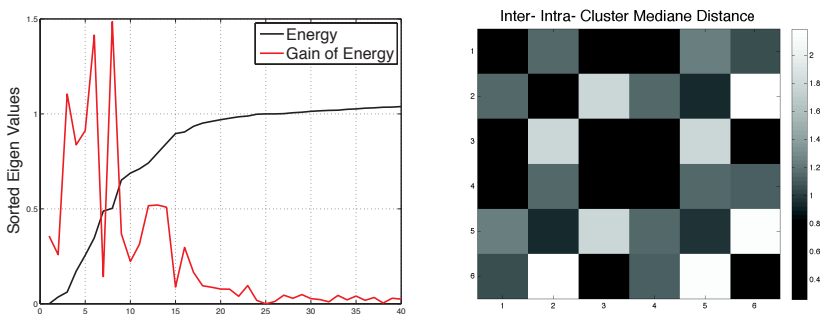

Figure 7. Spectral ${ }^{\text {Rank }}$ Clustering. Left: eigenvalues in ascending orders and successive differences (magnified by a factor 10). Right, Inter- and Intracluster distances.

the most significant (cf. Fig. 7, left). For our test datasets of 120 images, Fig. 7 (left) indicates local maxima for $K=3,6$ and 8 . Fig. 7 (right) shows the inter- and intra-cluster median distances obtained for $K=6$ clusters. Satisfactorily, it shows low intra-cluster median distances. It also shows that Clusters 1 and 4, Clusters2 and 5, Clusters 3 and 6, respectively, have low inter-cluster median distances. Interestingly, these pairwise groups of clusters quasi-exactly correspond to the clusters obtained at level $K=3$.

Making use of the documentation available for each sheet of paper enables us to establish the following constitution of the clusters:

Cluster 1 (26 samples, Half-Matte \& Chamois) gathers samples from one same paper sheet with chamois reflectance and 10 samples from the same packet with Half-Matte reflectance, respectively corresponding to Leonar (Manuf.) Rano Kraftig (Brand) \& Ilford (Manuf.) Plastika (Brand).

Cluster 4 (28 samples, Fine-Grained Luster) gathers samples from one same paper sheet and samples from the same packet, all with Lustre reflectance, and Fine-Grained texture, nicely gathering most of Kodak (Manuf.) Kodabromide (Brand) sheets, thus revealing the proximity between Kodabromide Sheet and Kodabromide packet.

Cluster 3 (24 samples, Smooth Glossy) gathers samples from one same paper sheet and samples from the same manufacturer, all with Glossy reflectance, and Smooth texture, nicely gathering most of Ilford \& Kodak (Manuf.) and Contact (Brand).

Cluster 6 (26 samples, Smooth Matte \& Semi-Matte)) gathers samples from one same paper sheet and samples from the same manufacturer, all with Matte or Semi-Matte reflectance, and Smooth or non-documented texture, gathering Kodack \& Dupond-defender Manufacturers.

Clusters 2 \& 5 (16 samples, Luster, Agfa)) gather samples from one same manufacturer Agfa, with Lustre reflectance. Though paper sheets in these clusters share the same reflectance as those in Cluster4, their texture appears different since being fine-grained in Cluster4 and non-documented for Clusters 2 \& 5. Interestingly, all paper sheets documented as manufacturer Agfa fall systematically in this cluster.

This study of the compositions of the clusters yields a number of interesting conclusions: i) samples from the same sheet are systematically in the same cluster; ii) samples from the same packet are systematically in the same cluster; iii) samples from 


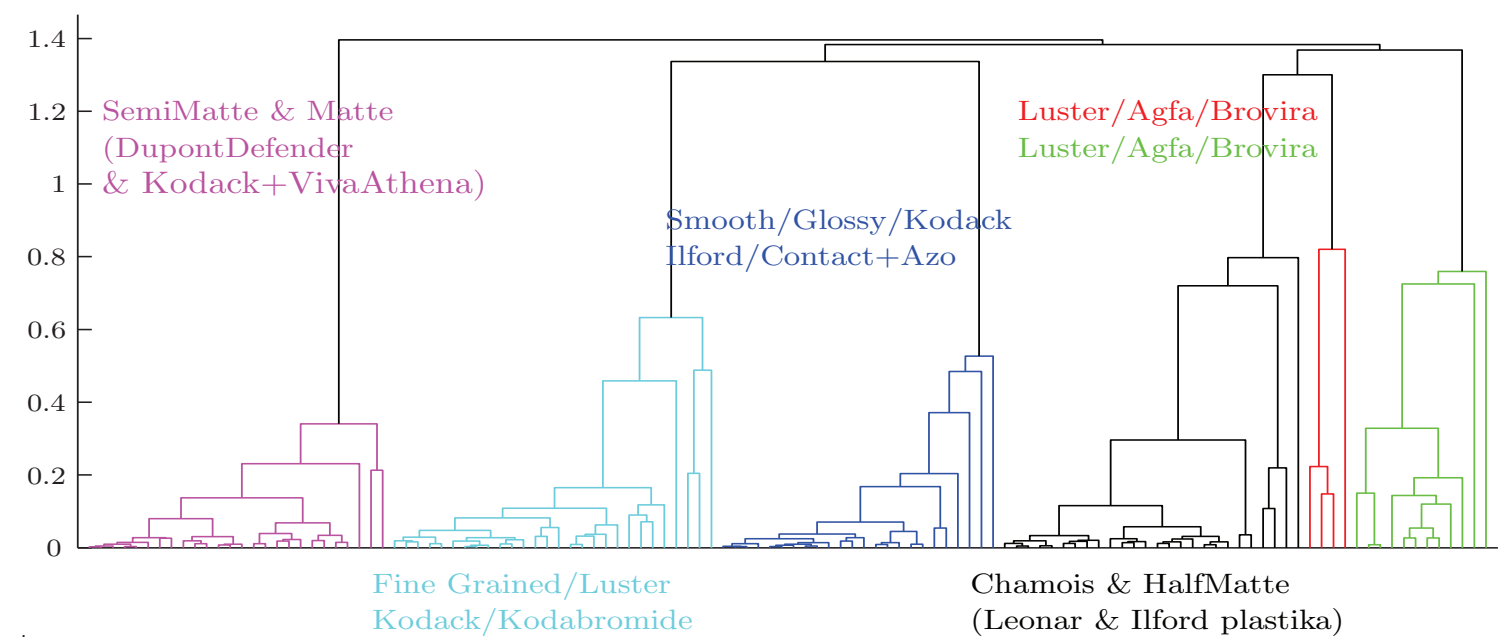

Figure 8. Classification summary as a dendrogram from Ascendant Hierarchical Clustering.

the same manufacturer essentially fall in the same cluster; iv) some paper sheets can obviously be associated to packet or manufacturer; v) Reflectance is the feature of the expert documentation that leads the clustering, with sub-clustering driven by texture; vi) it can be checked that for most of the 30 miscellaneous last samples, their reflectance match that of the cluster they fall in, a very encouraging result, showing the relevance of the automated classification procedure. These findings are summarized in the dendrogram reported in Fig. 8.

\section{ACKNOWLEDGMENT}

Work supported by ANR BLANC 2011 AMATIS BS0101102.

\section{REFERENCES}

[1] P. Abry, J. Coddington, I. Daubechies, E. Hendriks, S. Hughes, R. Johnson, and E. Postma. Special issue on image processing for digital art work guest editor's forewords. Signal Processing, Special issue, 93:525526, 2013.

[2] P. Abry, S. Jaffard, and H. Wendt. Bruegel's drawings under the multifractal microscope. In Proc. IEEE Int. Conf. Acoust., Speech, and Signal Proc. (ICASSP), Kyoto, Japan, 2012.

[3] P. Abry, H. Wendt, and S. Jaffard. When Van Gogh meets Mandelbrot: Multifractal classification of painting's texture. Signal Proc., 93(3, SI):554-572, 2013

[4] M. Barni, J.-A. Beraldin, C. Lahanier, and A. Piva. Signal processing in visual cultural heritage. Signal Processing Magazine, Special Issue, 25(4):10-13, 2008.

[5] C.L. Benhamou, S. Poupon, E. Lespessailles, S. Loiseau, R. Jennane, V. Siroux, W. J. Ohley, and L. Pothuaud. Fractal analysis of radiographic trabecular bone texture and bone mineral density: two complementary parameters related to osteoporotic fractures. J. Bone Miner. Res., 16(4):697-704, 2001.

[6] K. Deguchi. Two-dimensional auto-regressive model for analysis and synthesis of gray-level textures. Proc. of the First International Symposium for Science on Form, pages 441-449, 1986.

[7] R. A. DeVore, S. V. Konyagin, and V. N. Temlyakov. Hyperbolic wavelet approximation. Constructive Approximation, 14:1-26, 1998.

[8] S. Fortunato. Community detection in graphs. Physics Reports, 486(35):75-174, 2010.

[9] M. M. Galloway. Texture analysis using gray level run lengths. Computer Graphics Image Processing, 4:172-179, 1975.

[10] R. M. Haralick. Statistical and structural approaches to texture. Proc. of the IEEE, 67(5):786-804, 1979.
[11] T. Hastie, R. Tibshirani, and J. Friedman. The Elements of Statistical Learning. Springer Series in Statistics. Springer New York Inc., New York, NY, USA, 2001.

[12] S. Jaffard, P. Abry, and S. G. Roux. Function spaces vs. scaling functions: tools for image classification. Mathematical Image processing (Springer Proc. in Mathematics) M. Bergounioux ed., 5:1-39, 2011.

[13] C. R. Johnson, P. Messier, W. A. Sethares, A. G. Klein, C. Brown, P. Klausmeyer, P. Abry, S. Jaffard, H. Wendt, S. G. Roux, N. Pustelnik, N. van Noord, L. van der Maaten, E. Postma, J. Coddington, L. A. Daffner, H. Murata, H. Wilhelm, S. Wod, and M. Messier. Pursuing automated classification of historic photographic papers from raking light photomicrographs. Journal of the American Institute for Conservation, 53(3):159-170, 2014

[14] P. Kestener, J. Lina, P. Saint-Jean, and A. Arneodo. Wavelet-based multifractal formalism to assist in diagnosis in digitized mammograms. Image Analysis and Stereology, 20(3):169-175, 2004.

[15] R. Lopes and N. Betrouni. Fractal and multifractal analysis: A review. Medical Image Analysis, 13:634-649, 2009.

[16] N. Mallick, P. P. Cortet, S. Santucci, S. G. Roux, and L. Vanel. Discrepancy between subcritical and fast rupture roughness: A cumulant analysis. Phys. Rev. Lett., 98:255502, 2007.

[17] P. Messier. Photographic papers in the 20th century: Methodologies for research, authentication and dating. In Proceeding of FotoConservación, Logroño, Spain, 2011, 2011.

[18] P. Messier. The paul messier black and white photographic papers collection, 2013. http://paulmessier.com/pm/pdf/papers.

[19] P. Messier, C. R. Johnson, H. Wilhelm, W. A. Sethares, A. G. Klein, P. Abry, S. Jaffard, H. Wendt, S. G. Roux, N. Pustelnik, N. van Noord, L. van der Maaten, and E. Postma. Automated surface texture classification of inkjet and photographic media. In The 29th International Conference on Digital Printing Technologies, Seattle, USA, pages 8591. IS\&T: The Society for Imaging Science and Technology, 2013.

[20] D. Rockmore, J. Coddington, J. Elton, and Y. Wang. Multifractal analysis for Jackson Pollock. SPIE, pages 6810-13, 2008

[21] S. G. Roux, A. Arneodo, and N. Decoster. A wavelet-based method for multifractal image analysis. III. Applications to high-resolution satellite images of cloud structure. Eur. Phys. J. B, 15(4):765-786, 2000.

[22] S. G. Roux, M. Clausel, B. Vedel, S. Jaffard, and P. Abry. Self-Similar Anisotropic Texture Analysis: The Hyperbolic Wavelet Transform Contribution. IEEE Trans. on Image proc., 22(11):4353-4363, NOV 2013.

[23] Y. Stitou, F. Turcu, Y. Berthoumieu, and M. Najim. Three-dimensional textured image blocks model based on Wold decomposition. IEEE Trans. Signal Process., 55(7):3247-3261, Jul. 2007.

[24] M. Unser. Description statistique de la texture. $\mathrm{PhD}$ thesis, Ecole Polytechnique de Lausanne, Suisse, 1984.

[25] U. Von Luxburg. A tutorial on spectral clustering. Statistics and computing, 17(4):395-416, 2007.

[26] H. Wendt, S. G. Roux, P. Abry, and S. Jaffard. Wavelet leaders and bootstrap for multifractal analysis of images. Signal Processing, 89:1100-1114, 2009. 